Pacific

Journal of

Mathematics

A FREE ENTROPY DIMENSION LEMMA

KENLEY JUNG

Volume $211 \quad$ No. 2

October 2003 


\title{
A FREE ENTROPY DIMENSION LEMMA
}

\author{
KENLEY JunG \\ For Arlan Ramsay
}

\begin{abstract}
Suppose $M$ is a von Neumann algebra with normal, tracial state $\varphi$ and $\left\{a_{1}, \ldots, a_{n}\right\}$ is a set of self-adjoint elements in $M$. We provide an alternative uniform packing description of $\delta_{0}\left(a_{1}, \ldots, a_{n}\right)$, the modified free entropy dimension of $\left\{a_{1}, \ldots, a_{n}\right\}$.
\end{abstract}

In the attempt to understand the free group factors Voiculescu created a type of noncommutative probability theory. One facet of the theory involves free entropy and free entropy dimension, applications of which have answered some old operator algebra questions ([1] and [4]). Roughly speaking, given self-adjoint elements $a_{1}, \ldots, a_{n}$ in a von Neumann algebra $M$ with normal, tracial state $\varphi$ a matricial microstate for $\left\{a_{1}, \ldots, a_{n}\right\}$ is an $n$-tuple of self-adjoint $k \times k$ matrices which together with the normalized trace, approximate the algebraic behavior of the $a_{i}$ under $\varphi$. Taking a normalization of the logarithmic volume of such microstate sets followed by multiple limiting processes yields a number, $\chi\left(a_{1}, \ldots, a_{n}\right)$, called the free entropy of $\left\{a_{1}, \ldots, a_{n}\right\}$. One can think of free entropy as the logarithmic volume of the $n$-tuple. The (modified) free entropy dimension of $\left\{a_{1}, \ldots, a_{n}\right\}$ is

$$
\delta_{0}\left(a_{1}, \ldots, a_{n}\right)=n+\limsup _{\epsilon \rightarrow 0} \frac{\chi\left(a_{1}+\epsilon s_{1}, \ldots, a_{n}+\epsilon s_{n}: s_{1}, \ldots, s_{n}\right)}{|\log \epsilon|}
$$

where $\left\{s_{1}, \ldots, s_{n}\right\}$ is a semicircular family freely independent with respect to $\left\{a_{1}, \ldots, a_{n}\right\}$ and $\chi(:)$ is a technical modification of $\chi$ (see $\left.[4]\right)$.

Free entropy dimension was inspired by Minkowski dimension. Recall that for a subset $A \subset \mathbb{R}^{d}$ the (upper) Minkowski dimension of $A$ is

$$
d+\limsup _{\epsilon \rightarrow 0} \frac{\log \lambda\left(\mathcal{N}_{\epsilon}(A)\right)}{|\log \epsilon|}
$$

where $\lambda$ above denotes Lebesgue measure and $\mathcal{N}_{\epsilon}(A)$ is the $\epsilon$-neighborhood of $A$. Minkowski dimension has an equivalent formulation in terms of uniform packing dimension. The (upper) uniform packing dimension of $A$ is

$$
\limsup _{\epsilon \rightarrow 0} \frac{\log P_{\epsilon}(A)}{|\log \epsilon|}=\limsup _{\epsilon \rightarrow 0} \frac{\log K_{\epsilon}(A)}{|\log \epsilon|}
$$


where $A$ is endowed with the Euclidean metric, $P_{\epsilon}(A)$ is the maximum number of elements in a collection of mutually disjoint open $\epsilon$ balls of $A$, and $K_{\epsilon}(A)$ is the minimum number of open $\epsilon$ balls required to cover $A$ (the quantities above make sense in the setting of an arbitrary metric space). It is easy to see that the Minkowski dimension and the uniform packing dimension of $A$ are always equal.

In this paper we present a lemma which formulates a similar metric description of $\delta_{0}$ : Free entropy dimension can be described in terms of packing numbers with balls of equal radius.

The alternative description comes as no surprise in view of both the definition of $\delta_{0}$ and the techniques in estimations thereof. The proof follows the classical one with the addition of the properties of $\chi$ proven in $[\mathbf{3}]$ and the strengthened asymptotic freeness results of [5].

\section{Preliminaries.}

Throughout $M$ is a von Neumann algebra with normal, tracial state $\varphi$ and $\left\{a_{1}, \ldots, a_{n}\right\}$ is a set of self-adjoint elements in $M$. We use the symbols $\chi$ and $\delta_{0}$ to designate the same quantities introduced in [4]. $M_{k}^{\mathrm{sa}}(\mathbb{C})$ denotes the set of $k \times k$ self-adjoint complex matrices and $\left(M_{k}^{\mathrm{sa}}(\mathbb{C})\right)^{n}$ is the set of $n$-tuples with entries in $M_{k}^{\mathrm{sa}}(\mathbb{C}) \cdot \operatorname{tr}_{k}$ is the normalized trace on the $k \times k$ complex matrices. $\|\cdot\|_{2}$ is the inner product norm on $\left(M_{k}^{\mathrm{sa}}(\mathbb{C})\right)^{n}$ given by the formula $\left\|\left(x_{1}, \ldots, x_{n}\right)\right\|_{2}^{2}=\sum_{i=1}^{n} k \cdot \operatorname{tr}_{k}\left(x_{i}^{2}\right)$ and vol denotes Lebesgue measure with respect to the $\|\cdot\|_{2}$ norm. For any $k \in \mathbb{N}$ denote by $\rho_{k}$ the metric on $\left(M_{k}^{\mathrm{sa}}(\mathbb{C})\right)^{n}$ induced by the norm $k^{-\frac{1}{2}} \cdot\|\cdot\|_{2}$. For a metric space $(X, d)$ and $\epsilon>0$ write $P_{\epsilon}(X, d)$ for the maximum number of elements in a collection of mutually disjoint open $\epsilon$ balls of $X$ and $K_{\epsilon}(X, d)$ for the minimum number of open $\epsilon$ balls required to cover $X$. Observe that $P_{\epsilon}(X, d) \geq K_{2 \epsilon}(X, d) \geq$ $P_{4 \epsilon}(X, d)$. Finally for $S \subset X$ denote by $\mathcal{N}_{\epsilon}(S)$ the $\epsilon$-neighborhood of $S$.

\section{The lemma.}

Definition 2.1. For any $k, m \in \mathbb{N}$, and $R, \gamma, \epsilon>0$ define successively

$$
\begin{aligned}
\mathbb{P}_{\epsilon, R}\left(a_{1}, \ldots, a_{n} ; m, k, \gamma\right) & =P_{\epsilon}\left(\Gamma_{R}\left(a_{1}, \ldots, a_{n} ; m, k, \gamma\right), \rho_{k}\right), \\
\mathbb{P}_{\epsilon, R}\left(a_{1}, \ldots, a_{n} ; m, \gamma\right) & =\limsup _{k \rightarrow \infty} k^{-2} \cdot \log \left(\mathbb{P}_{\epsilon, R}\left(a_{1}, \ldots, a_{n} ; m, k, \gamma\right)\right), \\
\mathbb{P}_{\epsilon, R}\left(a_{1}, \ldots, a_{n}\right) & =\inf \left\{\mathbb{P}_{\epsilon, R}\left(a_{1}, \ldots, a_{n} ; m, \gamma\right): m \in \mathbb{N}, \gamma>0\right\}, \\
\mathbb{P}_{\epsilon}\left(a_{1}, \ldots, a_{n}\right) & =\sup _{R>0}\left\{\mathbb{P}_{\epsilon, R}\left(a_{1}, \ldots, a_{n}\right)\right\} .
\end{aligned}
$$

Remark. If $b_{1}, \ldots, b_{p} \in M$, then define $\mathbb{P}_{\epsilon}\left(a_{1}, \ldots, a_{n}: b_{1}, \ldots, b_{p}\right)$ to be the quantity obtained by replacing $\Gamma_{R}\left(a_{1}, \ldots, a_{n} ; m, k, \gamma\right)$ in the definition with $\Gamma_{R}\left(a_{1}, \ldots, a_{n}: b_{1}, \ldots, b_{p} ; m, k, \gamma\right)$. Similarly, we define $\mathbb{K}_{\epsilon}\left(a_{1}, \ldots, a_{n}\right)$ and 
all its associated quantities by replacing $P_{\epsilon}$ in the first line of Definition 2.1 with $K_{\epsilon}$. Define $\mathbb{K}_{\epsilon}\left(a_{1}, \ldots, a_{n}: b_{1}, \ldots, b_{p}\right)$ in the same way $\mathbb{P}_{\epsilon}\left(a_{1}, \ldots, a_{n}\right.$ : $\left.b_{1}, \ldots, b_{p}\right)$ was defined.

For any self-adjoint elements $h_{1}, \ldots, h_{n} \in M$ denote by $\chi\left(h_{1}, \ldots, h_{n}\right)$ the number obtained by replacing the lim sup in the definition of $\chi$ with lim inf . $\mathbb{P}_{\epsilon}(\cdot)$ being a normalized limiting process of the logarithmic microstate space packing numbers we observe just as in the classical case that:

Lemma 2.2. If $\left\{h_{1}, \ldots, h_{n}\right\}$ is a set of self-adjoint elements in $M$ which is freely independent with respect to $\left\{a_{1}, \ldots, a_{n}\right\}$ and $\underline{\chi}\left(h_{1}, \ldots, h_{n}\right)>-\infty$, then

$$
\begin{aligned}
& n+\limsup _{\epsilon \rightarrow 0} \frac{\chi\left(a_{1}+\epsilon h_{1}, \ldots, a_{n}+\epsilon h_{n}: h_{1}, \ldots, h_{n}\right)}{|\log \epsilon|} \\
& =\limsup _{\epsilon \rightarrow 0} \frac{\mathbb{K}_{\epsilon}\left(a_{1}, \ldots, a_{n}\right)}{|\log \epsilon|} \\
& =\limsup _{\epsilon \rightarrow 0} \frac{\mathbb{P}_{\epsilon}\left(a_{1}, \ldots, a_{n}\right)}{|\log \epsilon|} .
\end{aligned}
$$

Proof. Clearly it suffices to show equality of the first and last expressions above since $P_{\epsilon}(\cdot) \geq K_{2 \epsilon}(\cdot) \geq P_{4 \epsilon}(\cdot)$. Furthermore, we can assume that $\left\{a_{1}, \ldots, a_{n}\right\}$ has finite dimensional approximants since the equalities hold trivially otherwise. Set $C=\max \left\{\left\|h_{i}\right\|\right\}_{1 \leq i \leq n}+1$. First we show that the free entropy expression is greater than or equal to the $\mathbb{P}_{\epsilon}$ expression. Suppose $0<\epsilon<(C \sqrt{n})^{-1}, m \in \mathbb{N}$, with $m>n, 1>\gamma>0$, and $R>\max \left\{\left\|a_{i}\right\|\right\}_{1 \leq i \leq n}$.

Corollary 2.14 of [5] provides an $N \in \mathbb{N}$ such that if $k \geq N$ and $\sigma$ is a Radon probability measure on $\left(\left(M_{k}^{\mathrm{sa}}(\mathbb{C})\right)_{R+1}\right)^{2 n}$ (the subset of $\left(M_{k}^{\mathrm{sa}}(\mathbb{C})\right)^{2 n}$ consisting of $2 n$-tuples whose entries have operator norm no greater than $R+1)$ invariant under the $U_{k}$-action

$$
\left(\xi_{1}, \ldots, \xi_{n}, \eta_{1}, \ldots, \eta_{n}\right) \mapsto\left(\xi_{1}, \ldots, \xi_{n}, u \eta_{1} u^{*}, \ldots, u \eta_{n} u^{*}\right),
$$

then $\sigma\left(\omega_{k}\right)>\frac{1}{2}$ where $\omega_{k}$ is

$$
\begin{aligned}
\left\{\left(\xi_{1}, \ldots, \xi_{n}, \eta_{1}, \ldots, \eta_{n}\right) \in\left(\left(M_{k}^{\mathrm{sa}}(\mathbb{C})\right)_{R+1}\right)^{2 n}:\left\{\xi_{1}, \ldots, \xi_{n}\right\}\right. \\
\text { and } \left.\left\{\eta_{1}, \ldots, \eta_{n}\right\} \text { are }\left(m, \gamma / 4^{m}\right) \text {-free }\right\} .
\end{aligned}
$$

With respect to the $\rho_{k}$ metric for each $k$ find a collection of mutually disjoint open $C \epsilon \sqrt{n}$ balls of $\Gamma_{R}\left(a_{1}, \ldots, a_{n} ; m, k, \gamma /(8(R+2))^{m}\right)$ of maximum cardinality and denote the centers of these balls by $\left\langle\left(x_{1 j}^{(k)}, \ldots, x_{n j}^{(k)}\right)\right\rangle_{j \in S_{k}}$. Let $\mu_{k}$ be the uniform atomic probability measure supported on the centers of these balls and let $\nu_{k}$ be the probability measure obtained by restricting vol to $\Gamma_{C \epsilon}\left(\epsilon h_{1}, \ldots, \epsilon h_{n} ; m, k, \gamma / 8^{m}\right)$ and normalizing appropriately. Then $\mu_{k} \times \nu_{k}$ 
is a Radon probability measure on $\left(\left(M_{k}^{\mathrm{sa}}(\mathbb{C})\right)_{R+1}\right)^{2 n}$ invariant under the $U_{k}$-action described above. So for $k \geq N\left(\mu_{k} \times \nu_{k}\right)\left(\omega_{k}\right)>\frac{1}{2}$.

For $k \in \mathbb{N}$ and $j \in S_{k}$ define $F_{j k}$ to be the set of all $\left(y_{1}, \ldots, y_{n}\right) \in$ $\Gamma_{C \epsilon}\left(\epsilon h_{1}, \ldots, \epsilon h_{n} ; m, k, \gamma / 8^{m}\right)$ such that $\left(y_{1}, \ldots, y_{n}\right)$ and $\left(x_{1 j}^{(k)}, \ldots, x_{n j}^{(k)}\right)$ are $\left(m, \frac{\gamma}{4^{m}}\right)$-free.

$$
\begin{aligned}
\frac{1}{2}<\left(\mu_{k} \times \nu_{k}\right)\left(\omega_{k}\right) & =\sum_{j \in S_{k}} \frac{1}{\left|S_{k}\right|} \cdot \nu_{k}\left(F_{j k}\right) \\
& =\sum_{j \in S_{k}} \frac{1}{\left|S_{k}\right|} \cdot \frac{\operatorname{vol}\left(F_{j k}\right)}{\operatorname{vol}\left(\Gamma_{C \epsilon}\left(\epsilon h_{1}, \ldots, \epsilon h_{n} ; m, k, \gamma / 8^{m}\right)\right)} .
\end{aligned}
$$

It follows that for $k \geq N$

$$
\frac{1}{2} \cdot\left|S_{k}\right| \cdot \operatorname{vol}\left(\Gamma_{C \epsilon}\left(\epsilon h_{1}, \ldots, \epsilon h_{n} ; m, k, \gamma / 8^{m}\right)\right)<\sum_{j \in S_{k}} \operatorname{vol}\left(F_{j k}\right) .
$$

Set $E_{j k}=\left(x_{1 j}^{(k)}, \ldots, x_{n j}^{(k)}\right)+F_{j k} . F_{j k}$ is a set contained in the open ball of $\rho_{k}$ radius $C \epsilon \sqrt{n}$ centered at $(0, \ldots, 0)$. Thus $\left\langle E_{j k}\right\rangle_{j \in S_{k}}$ is a collection of mutually disjoint sets. So

$$
\bigsqcup_{j \in S_{k}} E_{j k} \subset \Gamma_{R+1}\left(a_{1}+\epsilon h_{1}, \ldots, a_{n}+\epsilon h_{n}: \epsilon h_{1}, \ldots, \epsilon h_{n} ; m, k, \gamma\right)
$$

Thus, for any $(C \sqrt{n})^{-1}>\epsilon>0, m \in \mathbb{N}$ sufficiently large, and $1>\gamma>0$

$$
\begin{aligned}
& \chi_{R+1}\left(a_{1}+\epsilon h_{1}, \ldots, a_{n}+\epsilon h_{n}: \epsilon h_{1}, \ldots, \epsilon h_{n} ; m, \gamma\right) \\
& \geq \limsup _{k \rightarrow \infty}\left(k^{-2} \cdot \log \left(\operatorname{vol}\left(\bigsqcup_{j \in S_{k}} E_{j k}\right)\right)+\frac{n}{2} \cdot \log k\right) \\
& \geq \underset{k \rightarrow \infty}{\limsup _{k \rightarrow \infty}}\left[k^{-2} \cdot \log \left(\frac{1}{2} \cdot\left|S_{k}\right| \cdot \operatorname{vol}\left(\Gamma_{C \epsilon}\left(\epsilon h_{1}, \ldots, \epsilon h_{n} ; m, k, \gamma / 8^{m}\right)\right)\right)\right. \\
& \left.\quad+\frac{n}{2} \log k\right] \\
& \geq \limsup _{k \rightarrow \infty}\left[k^{-2} \cdot \log \left(\left|S_{k}\right|\right)\right] \\
& \quad+\liminf _{k \rightarrow \infty}\left[k^{-2} \cdot \log \left(\operatorname{vol}\left(\Gamma_{C \epsilon}\left(\epsilon h_{1}, \ldots, \epsilon h_{n} ; m, k, \gamma / 8^{m}\right)\right)\right)+\frac{n}{2} \cdot \log k\right] \\
& \geq \mathbb{P}_{C \epsilon \sqrt{n}, R+1}\left(a_{1}, \ldots, a_{n} ; m, \gamma /(8(R+2))^{m}\right)+\underline{\chi C \epsilon}\left(\epsilon h_{1}, \ldots, \epsilon h_{n}\right) \\
& \geq \mathbb{P}_{C \epsilon \sqrt{n}, R+1}\left(a_{1}, \ldots, a_{n}\right)+n \log \epsilon+\underline{\chi}\left(h_{1}, \ldots, h_{n}\right) .
\end{aligned}
$$


By the chain of inequalities of the preceding paragraph it follows that

$$
\begin{aligned}
& \chi\left(a_{1}+\epsilon h_{1}, \ldots, a_{n}+\epsilon h_{n}: h_{1}, \ldots, h_{n}\right) \\
& =\chi\left(a_{1}+\epsilon h_{1}, \ldots, a_{n}+\epsilon h_{n}: \epsilon h_{1}, \ldots, \epsilon h_{n}\right) \\
& \geq \mathbb{P}_{C \epsilon \sqrt{n}, R+1}\left(a_{1}, \ldots, a_{n}\right)+n \log \epsilon+\underline{\chi}\left(h_{1}, \ldots, h_{n}\right) .
\end{aligned}
$$

This being true for $R$ sufficiently large

$$
\begin{aligned}
& \chi\left(a_{1}+\epsilon h_{1}, \ldots, a_{n}+\epsilon h_{n}: h_{1}, \ldots, h_{n}\right) \\
& \geq \mathbb{P}_{C \epsilon \sqrt{n}}\left(a_{1}, \ldots, a_{n}\right)+n \log \epsilon+\underline{\chi}\left(h_{1}, \ldots, h_{n}\right) .
\end{aligned}
$$

Dividing by $|\log \epsilon|$ on both sides, taking a $\lim \sup$ as $\epsilon \rightarrow 0$, and adding $n$ to both ends of the inequality above yields

$$
\begin{aligned}
& n+\limsup _{\epsilon \rightarrow 0} \frac{\chi\left(a_{1}+\epsilon h_{1}, \ldots, a_{n}+\epsilon h_{n}: h_{1}, \ldots, h_{n}\right)}{|\log \epsilon|} \\
& \geq \limsup _{\epsilon \rightarrow 0} \frac{\mathbb{P}_{C \epsilon \sqrt{n}}\left(a_{1}, \ldots, a_{n}\right)}{|\log \epsilon|} \\
& =\limsup _{\epsilon \rightarrow 0} \frac{\mathbb{P}_{\epsilon}\left(a_{1}, \ldots, a_{n}\right)}{|\log \epsilon|} .
\end{aligned}
$$

For the reverse inequality suppose $2 \leq m \in \mathbb{N}$ and $\frac{1}{2(C+1)}>\epsilon>\sqrt{\gamma}>$ $0, R>\max _{1 \leq j \leq n}\left\{\left\|a_{j}\right\|\right\}$. For each $k \in \mathbb{N}$ find an packing by open $\rho_{k} \epsilon$-balls of $\Gamma_{R+1}\left(a_{1}, \ldots, a_{n} ; m, k, \gamma\right)$ with maximum cardinality. Denote the set of centers of these balls by $\Omega_{k}$. Clearly

$$
\begin{aligned}
& \Gamma_{R+\frac{1}{2}, \frac{1}{2}}\left(a_{1}+\epsilon h_{1}, \ldots, a_{n}+\epsilon h_{n}: \epsilon h_{1}, \ldots, \epsilon h_{n} ; m, k, \frac{\gamma}{2^{m}}\right) \\
& \subset \mathcal{N}_{2 C \epsilon \sqrt{n}}\left(\Gamma_{R+1}\left(a_{1}, \ldots, a_{n} ; m, k, \gamma\right)\right) \\
& \subset \mathcal{N}_{4 C \epsilon \sqrt{n}}\left(\Omega_{k}\right)
\end{aligned}
$$

where $\Gamma_{r+\frac{1}{2}, \frac{1}{2}}(\cdot)$ denotes the microstate space of $2 n$-tuples such that the first $n$ entries have operator norms no larger than $r+\frac{1}{2}$ and the last $n$ entries have operator norms no larger than $\frac{1}{2}$ (see [4] for this technical modification). $\mathcal{N}_{\epsilon}$ is taken with respect to the metric space $\left(M_{k}^{\mathrm{sa}}(\mathbb{C})\right)^{n}$ with the $\rho_{k}$ metric. It follows that $\chi_{R+\frac{1}{2}, \frac{1}{2}}\left(a_{1}+\epsilon h_{1}, \ldots, a_{n}+\epsilon h_{n}: \epsilon h_{1}, \ldots, \epsilon h_{n} ; m, \frac{\gamma}{2^{m}}\right)$ is dominated by

$$
\begin{aligned}
& \limsup _{k \rightarrow \infty}\left[k^{-2} \cdot \log \left(\operatorname{vol}\left(\mathcal{N}_{4 C \epsilon \sqrt{n}}\left(\Omega_{k}\right)\right)\right)+\frac{n}{2} \cdot \log k\right] \\
& \leq \limsup _{k \rightarrow \infty}\left[k^{-2} \cdot \log \left(\left|\Omega_{k}\right| \cdot \frac{\pi^{\frac{n k^{2}}{2}} \cdot(4 C \epsilon \sqrt{n k})^{n k^{2}}}{\Gamma\left(\frac{n k^{2}}{2}+1\right)}\right)+\frac{n}{2} \cdot \log k\right]
\end{aligned}
$$




$$
\begin{aligned}
\leq & \limsup _{k \rightarrow \infty} k^{-2} \cdot \log \left(\left|\Omega_{k}\right|\right) \\
& +\limsup _{k \rightarrow \infty}\left[n \log (4 C \epsilon \sqrt{n k \pi})-k^{-2} \cdot \log \left(\frac{n k^{2}}{2 e}\right)^{\frac{n k^{2}}{2}}+\frac{n}{2} \cdot \log k\right] \\
= & \underset{k \rightarrow \infty}{\limsup } k^{-2} \cdot \log \left(\left|\Omega_{k}\right|\right) \\
& +\limsup _{k \rightarrow \infty}\left(n \log (4 C \epsilon \sqrt{n \pi})-n \log \left(\frac{k \sqrt{n}}{\sqrt{2 e}}\right)+n \log k\right) \\
= & \limsup _{k \rightarrow \infty} k^{-2} \cdot \log \left(\left|\Omega_{k}\right|\right)+n \log (4 C \epsilon \sqrt{2 \pi e}) \\
= & \mathbb{P}_{\epsilon, R+1}\left(a_{1}, \ldots, a_{n} ; m, \gamma\right)+n \log (4 C \epsilon \sqrt{2 \pi e}) .
\end{aligned}
$$

This being true for any $2 \leq m \in \mathbb{N}, \frac{1}{2(R+1)}>\epsilon>\sqrt{\gamma}>0$, and $R>$ $\max _{1 \leq j \leq n}\left\{\left\|a_{j}\right\|\right\}$ it follows that for sufficiently small $\epsilon>0$

$$
\begin{aligned}
& \chi\left(a_{1}+\epsilon h_{1}, \ldots, a_{n}+\epsilon h_{n}: h_{1}, \ldots, h_{n}\right) \\
& =\chi_{R+\frac{1}{2}, \frac{1}{2}}\left(a_{1}+\epsilon h_{1}, \ldots, a_{n}+\epsilon h_{n}: \epsilon h_{1}, \ldots, \epsilon h_{n}\right) \\
& \leq \mathbb{P}_{\epsilon}\left(a_{1}, \ldots, a_{n}\right)+n \log \epsilon+n \log (4 C \sqrt{2 \pi e})
\end{aligned}
$$

Dividing by $|\log \epsilon|$, taking a $\lim \sup$ as $\epsilon \rightarrow 0$, and adding $n$ to both sides of the inequality above yields

$$
n+\limsup _{\epsilon \rightarrow 0} \frac{\chi\left(a_{1}+\epsilon h_{1}, \ldots, a_{n}+\epsilon h_{n}: h_{1}, \ldots, h_{n}\right)}{|\log \epsilon|} \leq \limsup _{\epsilon \rightarrow 0} \frac{\mathbb{P}_{\epsilon}\left(a_{1}, \ldots, a_{n}\right)}{|\log \epsilon|}
$$

Remark 2.3. Suppose $b_{1}, \ldots, b_{p}$ are contained in the strongly closed algebra generated by the $a_{i}$ and $R>0$ is strictly greater than the operator norm of any $a_{i}$ or $b_{j}$. The proof shows that the quanitity

$$
\limsup _{\epsilon \rightarrow 0} \frac{\mathbb{K}_{\epsilon, R}\left(a_{1}, \ldots, a_{n}: b_{1}, \ldots, b_{p}\right)}{|\log \epsilon|}=\limsup _{\epsilon \rightarrow 0} \frac{\mathbb{P}_{\epsilon, R}\left(a_{1}, \ldots, a_{n}: b_{1}, \ldots, b_{p}\right)}{|\log \epsilon|}
$$

equals

$$
n+\limsup _{\epsilon \rightarrow 0} \frac{\chi\left(a_{1}+\epsilon h_{1}, \ldots, a_{n}+\epsilon h_{n}: h_{1}, \ldots, h_{n}\right)}{|\log \epsilon|} .
$$

Recall that by [3] and [5] if $\left\{s_{1}, \ldots, s_{n}\right\}$ is a free semicircular family, then $\chi\left(s_{1}, \ldots, s_{n}\right)=\underline{\chi}\left(s_{1}, \ldots, s_{n}\right)>-\infty$. Thus we have by the lemma:

\section{Corollary 2.4.}

$$
\delta_{0}\left(a_{1}, \ldots, a_{n}\right)=\limsup _{\epsilon \rightarrow 0} \frac{\mathbb{P}_{\epsilon}\left(a_{1}, \ldots, a_{n}\right)}{|\log \epsilon|}=\limsup _{\epsilon \rightarrow 0} \frac{\mathbb{K}_{\epsilon}\left(a_{1}, \ldots, a_{n}\right)}{|\log \epsilon|} .
$$


Both descriptions of $\delta_{0}$, either in terms of volumes of $\epsilon$-neighborhoods or in terms of packing numbers, can be useful. In the presence of freeness or in the situation with one random variable it is fruitful to use the $\epsilon$-neighborhood description as Voiculescu did $([\mathbf{3}])$. On the other hand when computing $\delta_{0}$ in some examples it is convenient to use the uniform packing description and this was the implicit attitude taken towards $\delta_{0}$ in [2]. The packing formulation also comes in handy when proving formulas for generators of $M$ when $M$ has a simple algebraic decomposition into a tensor product of a von Neumann algebra $N$ with the $k \times k$ matrices or into a direct sum of algebras.

\section{References}

[1] L. Ge, Applications of free entropy to finite von Neumann algebras, II, Ann. of Math., 147 (1998), 143-157, MR 99c:46068, Zbl 0924.46050.

[2] K. Jung, The free entropy dimension of hyperfinite von Neumann algebras, to appear in Trans. Amer. Math. Soc.

[3] D. Voiculescu, The Analogues of entropy and of Fisher's information measure in free probability theory, II, Inventiones mathematicae, 118 (1994), 411-440, MR 96a:4611, Zbl 0820.60001.

[4] - The analogues of entropy and of Fisher's information measure in free probability theory III: The absence of Cartan subalgebras, Geom. Funct. Anal., 6(1) (1996), 172-199, MR 96m:46119, Zbl 0856.60012.

[5] _ A strengthened asymptotic freeness result for random matrices with applications to free entropy, Internat. Math. Res. Notices, 1 (1998), 41-64, MR 2000d:46080, Zbl 0895.60004.

Received August 28, 2002 and revised October 23, 2002. This research was supported by the NSF Graduate Fellowship Program.

Department of Mathematics

UNIVERSiTy OF CALIFORNIA

BERKELEy, CA 94720-3840

E-mail address: factor@math.berkeley.edu 